\title{
Predicting functional associations from metabolism using bi-partite network algorithms
}

Balaji Veeramani, ${ }^{1,2}$, Joel S Bader ${ }^{1,2^{*}}$

\begin{abstract}
Background: Metabolic reconstructions contain detailed information about metabolic enzymes and their reactants and products. These networks can be used to infer functional associations between metabolic enzymes. Many methods are based on the number of metabolites shared by two enzymes, or the shortest path between two enzymes. Metabolite sharing can miss associations between non-consecutive enzymes in a serial pathway, and shortest-path algorithms are sensitive to high-degree metabolites such as water and ATP that create connections between enzymes with little functional similarity.

Results: We present new, fast methods to infer functional associations in metabolic networks. A local method, the degree-corrected Poisson score, is based only on the metabolites shared by two enzymes, but uses the known metabolite degree distribution. A global method, based on graph diffusion kernels, predicts associations between enzymes that do not share metabolites. Both methods are robust to high-degree metabolites. They out-perform previous methods in predicting shared Gene Ontology (GO) annotations and in predicting experimentally observed synthetic lethal genetic interactions. Including cellular compartment information improves GO annotation predictions but degrades synthetic lethal interaction prediction. These new methods perform nearly as well as computationally demanding methods based on flux balance analysis.

Conclusions: We present fast, accurate methods to predict functional associations from metabolic networks. Biological significance is demonstrated by identifying enzymes whose strong metabolic correlations are missed by conventional annotations in $\mathrm{GO}$, most often enzymes involved in transport vs. synthesis of the same metabolite or other enzyme pairs that share a metabolite but are separated by conventional pathway boundaries. More generally, the methods described here may be valuable for analyzing other types of networks with long-tailed degree distributions and high-degree hubs.
\end{abstract}

\section{Background}

High quality metabolic reconstructions are available for many organisms and provide a rich scaffold for interpreting data from high-throughput biological experiments. The topology of a metabolic network, defined by connections between enzymes and metabolites, can be used to predict genetic interactions, transcriptional correlations and disease co-morbidity [1-3].

Previous studies have used the topology of the metabolic network to predict co-expression of transcripts for yeast metabolic enzymes [4]. This study first removed high-degree metabolites from the bipartite metabolic network, generated an enzyme-only network by

\footnotetext{
* Correspondence: joel.bader@jhu.edu

'Department of Biomedical Engineering, Johns Hopkins University, Baltimore,
} MD21218, USA connecting enzymes that shared at least 1 remaining metabolite, and calculated the shortest-path distance between all pairs of enzymes. Shorter distances were correlated with stronger co-expression. Similar procedures, also excluding high-degree metabolites from consideration, were used recently in a study linking diseases to metabolic enzymes [3].

Methods that involve calculation of optimal fluxes subject to constraints, such as flux coupling [5], have performed better than local topological metrics based on shared neighbours in predicting transcript co-expression. Flux coupling methods are much more computationally expensive than topological analysis, however. Furthermore, flux coupling methods suffer from the disadvantage that reactions with small flux values (and hence the enzymes involved in those reactions) are

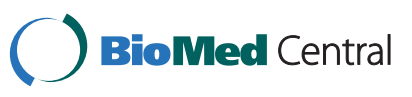


typically removed from the network. This is a problem if an enzyme of interest is removed from the network based on low reaction flux.

Our goal is to provide improved topological measures for enzyme functional associations from metabolic networks without the need for expensive calculations of optimal fluxes or sampling over feasible flux space. The motivation of our approach is that methods that count shared metabolites, or methods that generate a p-value for shared metabolites based on a hypergeometric distribution, essentially assume a flat degree distribution for metabolites. High-degree metabolites violate the assumption of a flat degree distribution, and the hypergeometric distribution is inappropriate for calculating p-values for metabolite sharing. Randomization methods based on rewiring, which maintain the observed degree distribution, are robust to high-degree metabolites but unfortunately are computationally expensive.

In this work, we provide a series of scores that are the Bayesian equivalent of the hypergeometric distribution, but adjusted for the known metabolite degree distributions. These scores are fast to calculate, essentially no more expensive than a hypergeometric p-value, and much faster than any methods that require rewiring permutations, flux sampling, or flux optimization. Results from applying these methods to metabolic networks in yeast demonstrate performance better than previous methods based on local connectedness. The results also reveal functional associations that are not captured by conventional metabolic pathway definitions, but which are inherent in the network structure.

\section{Results}

\section{Overview}

Metabolic networks can be represented as bipartite graphs with edges between enzymes and metabolites. An enzyme can use a metabolite in multiple unique reactions involving distinct subsets of other metabolites, and the number of unique reactions defines an integervalued edge weight.

A graphical overview shows how metabolic network information is incorporated to yield increasingly sophisticated models (Figure 1). The models discussed here are all designed to rank pairs of enzymes for functional association. Methods termed "Local" are capable only of producing rankings for enzymes directly connected by at least one metabolite. The raw number of shared metabolites $[3,6]$ and the hypergeometric distribution that corrects the shared count for the enzyme degree (Figure 1A) are both local methods.

In this work, we introduce a more sophisticated local method that also corrects for metabolite degree, discounting the contribution of highly connected metabolites like water, protons, and ATP (Figure 1B). Our new local methods are motivated by Bayesian model selection using the log-likelihood ratio of a null model (random connectivity between enzymes and metabolites) to an alternative model. The number of shared metabolites is modelled as a Poisson distribution for both the null and alternative models. For the alternative model, the Poisson parameter is the maximum likelihood estimate for the observed network, which is the observed number of shared metabolites. For the null model, the Poisson parameter is estimated from a random network model (virtually identical to the leading contribution to the hypergeometric distribution). We present results for an improved Poisson model that uses knowledge of the observed metabolite degree distribution.

Methods termed "Global" are capable of generating rankings for enzymes that are not directly connected by metabolites by using the full network topology. Examples are shortest paths and the more robust graph diffusion kernel (GDK) (Figure 1C). GDKs are non-local in that they sample over all paths between two enzymes, rather than just the shortest paths defined by shared metabolites. GDKs have been successfully applied to functional inference in metabolic networks [7]. Recently parity-specific kernels been used to analyze genetic interaction networks [8]. We also used a method based on the Pearson correlation of the weighted metaboliteenzyme edge connectivity structure between two enzymes.

Yet more elaborate flux balance analysis methods sample flux states that are feasible under steady-state constraints. Flux correlations can then be used to rank enzyme pairs for functional associations (Figure 1D). Other flux balance methods have generated functional associations by predicting synergistic or buffering epistatic interactions for deleting pairs of enzymes from the network.

\section{Performance of local methods}

Performance is assessed primarily by the ability to predict synthetic lethal genetic interactions between metabolic enzymes, and secondarily by the ability to identify classes of enzymes with similar Gene Ontology (GO) annotations. The synthetic lethal interactions provide a direct link to testable experiments. The database annotations are not necessarily testable, but instead show whether inference from computational models is consistent with known biology.

We generated rank ordered lists of enzyme pairs based on the local methods. Performance was assessed from the receiver operating characteristic (ROC) curve using the area under the curve (AUC), and from the precision-recall $(\mathrm{PR})$ curve using the maximum F-score, the harmonic mean of precision and recall. Known positives were taken from experimentally reported synthetic 
lethal/growth defect interactions recorded in the BioGRID database. There were 170 growth defect/lethal interactions in which both genes involved were part of the metabolic network model. Known negatives were defined as gene pairs where each gene has at least one synthetic lethal interaction, and one of the two has at least 5 synthetic lethal interactions as a query in a highthroughput screen, to exclude pairs that might not have been tested experimentally.

Performance metrics for the local methods in predicting synthetic lethal genetic interactions are the AUC and F-score (Figure 2). Raw counts of shared metabolites perform the worst. Thresholding the network to remove high-degree metabolites introduces changes in the performance only slightly, and results remain inferior to global methods described below (Figure 3, see [Additional file 1] for details of metabolites removed). The methods that account for the degree of an enzyme, but assume a flat metabolite degree distribution, improve on the raw counts. The new method, which accounts for the metabolite degree as well, performs at least as well as previous methods, and the precision degrades the most slowly of all methods at higher recall.

\section{Performance of global topology methods}

We then compared the performance of the best local method (Poisson score using metabolite degree distribution) with methods that use global topology and incorporate local edge weights (Figure 4). Global topology is incorporated using a graph diffusion kernel that sums paths of all lengths between enzyme pairs, rather than just the number of shortest paths involving shared metabolites. Local weights for enzyme-metabolite edges are taken from the integer number of unique reactions involving each enzyme-metabolite pair; these weighted edges were then used to construct a graph diffusion kernel. 


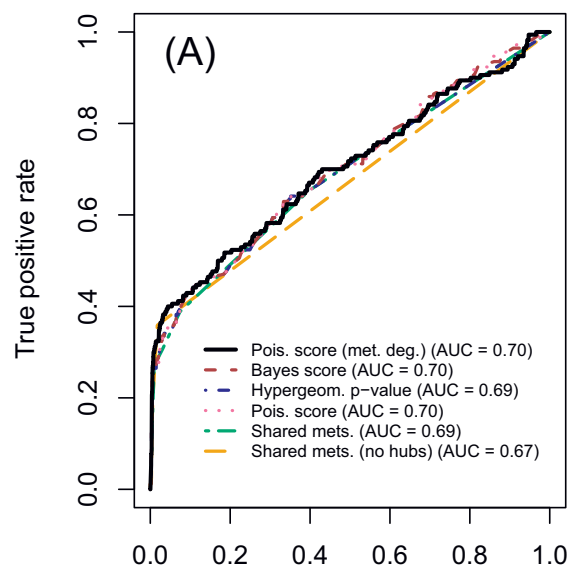

False positive rate

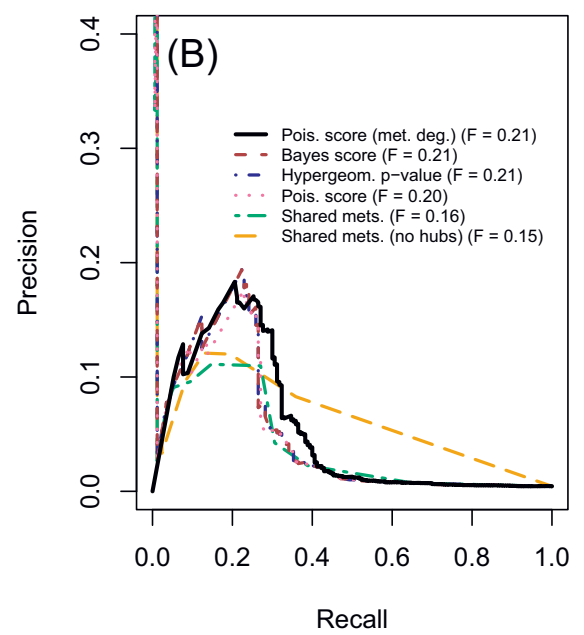

Figure 2 Performance comparison, local methods. Methods are listed, from bottom to top, roughly in order of improving performance in predicting synthetic lethal genetic interactions. Shared metabolites indicates raw counts of shared metabolites, and "no hubs" indicates that the most highly connected metabolites were removed before calculating sharing. The Poisson score, hypergeometric p-value, and Bayes score are based on the number of shared metabolites, but do not use the full metabolite degree distribution. The method "Poisson score (met. deg.)" uses the metabolite degree distribution for an additional correction. Performance is assessed using synthetic lethal gene pairs as known positives, and non-synthetic lethal pairs as known negatives. (A) Receiver operator characteristic (ROC) curve. (B) Precision-recall (PR) curve.
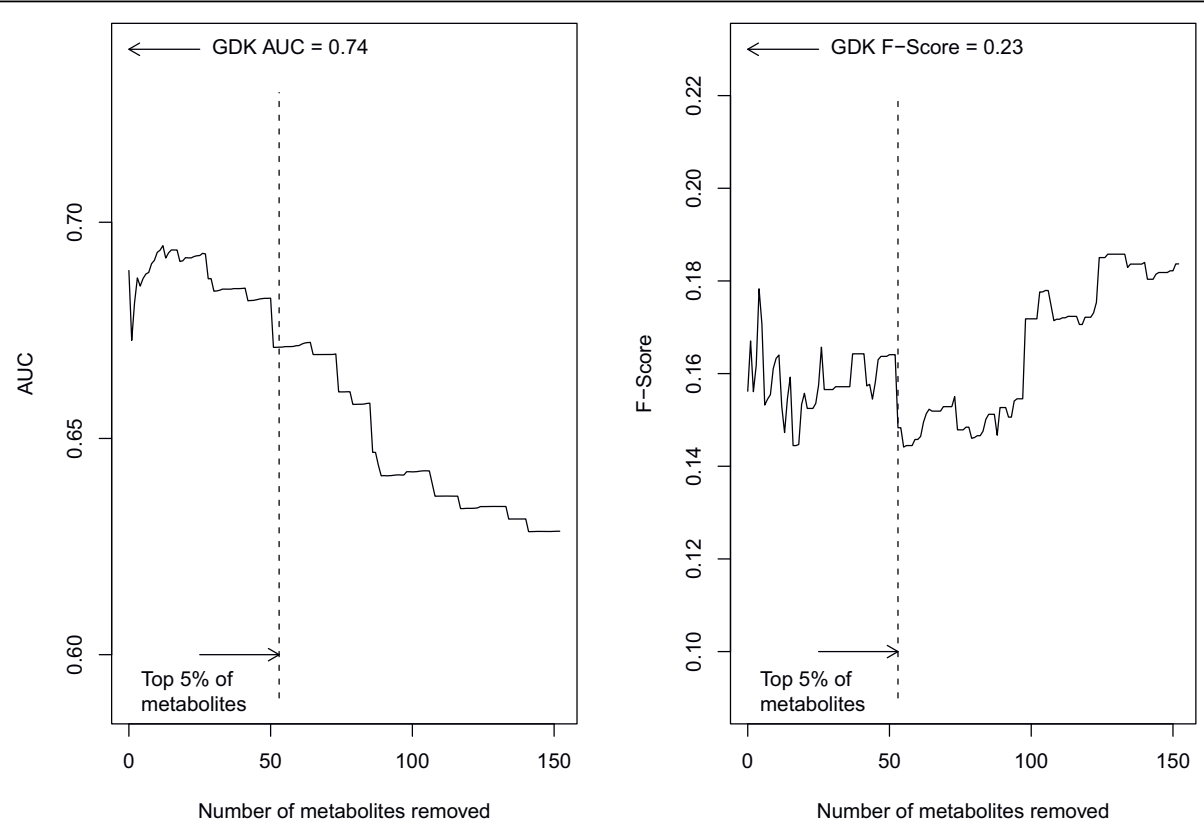

Figure 3 Performance of raw neighbour count with varying threshold for removing high-degree metabolites. The degree of each metabolite (the number of reactions it participates in as reactant or product) was calculated, and metabolites were progressively removed. After each removal, the performance of the raw neighbour count for predicting synthetic lethal interactions was assessed by the area under the receiver operating characteristic curve (AUC, panel A) and by the F-Score, defined the maximum harmonic mean of precision and recall along the precision-recall curve (F-Score, panel B). The results in the main text used a nominal threshold of the top 5\% of all metabolites, corresponding to 53 metabolites with degree $\geq 13$ of the entire set of 1061 metabolites. The results using a graph diffusion kernel (GDK) are shown to be superior to the raw neighbour regardless of the threshold used. 

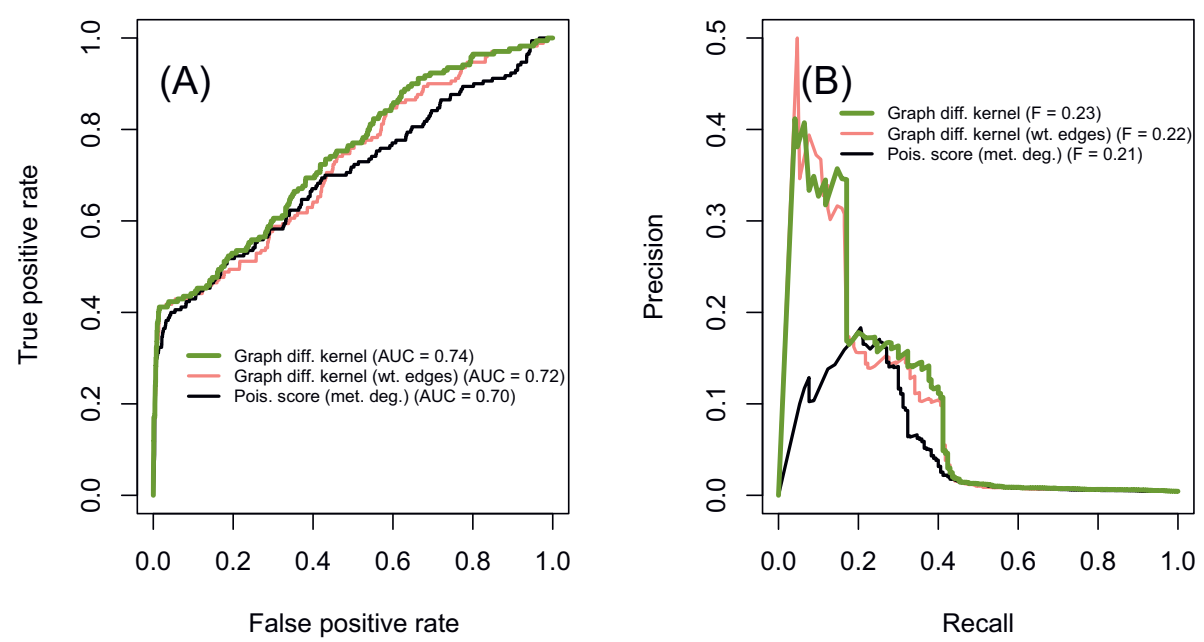

Figure 4 Performance comparison, local vs. global methods. The best local method, the Poisson score incorporating metabolite degree, is compared with global methods based on graph diffusion with and without edge weights accounting for multiple reactions for each enzyme. The global methods perform better, and weighted and unweighted diffusion kernels have equivalent performance. (A) ROC curve. (B) PR curve.

Overall, incorporating global information through the graph diffusion kernel improves the performance in identifying synthetic lethal pairs. Adding edge weights to the graph diffusion kernel does not appear to improve performance. Further comparisons with the graph diffusion kernel use the unweighted model only, as it is simpler.

\section{Global topology with metabolic constraints}

We next considered possible improvements that use the knowledge that the network edges represent a flux balance model for metabolism. While others have investigated models that investigate the robustness of metabolism to pairwise gene deletions [9], correlations of fluxes through enzymes provide improved predictions of genetic interactions [2]. We therefore used the flux sampling approach to calculate enzyme correlations, whose absolute values were used to rank enzyme pairs. The flux sampling excludes reactions with negligible flux, which reduces the model to 477 metabolites, 582 reactions and 469 enzymes and reduces the known positive pairs to 69 genetic interactions.

Comparison of the flux sampling method to the best local and global topology methods considers only the enzymes present in the reduced model (Figure 5). The flux sampling method has the best precision in the high-recall region. Enzyme pairs that are used in exactly the same reactions, or which are coupled in an obligate serial pathway, have a flux correlation of 1 . These pairs are all ranked identically by the flux sampling method, but may be ranked in a definite order by the local and global topology methods. In the low-recall region, consequently, the local and global methods appear to provide improved precision over the flux sampling approach.

The epistatic estimates, obtained from previous work [9], do not perform as well in predicting synthetic lethality.

\section{Compartmentalized metabolism}

Reactions in the metabolic network are localized to specific compartments (cytoplasm, mitochondria, extracellular, peroxisome, nucleus, Golgi apparatus, endoplasmic reticulum and vacuole). Of the total 1266 metabolic reactions in the model, $47 \%$ are entirely contained in the cytosol, roughly $10 \%$ are either mitochondrial or extracellular, and 24\% couple metabolites from different compartments (Table 1). If one metabolite appears in two different compartments, it is represented as two unique metabolites; enzymes in different compartments that process this metabolite are not scored as sharing it, reducing the ability to detect functional associations for enzymes in different compartments. On the other hand, functional association as measured by synthetic lethality is roughly $3 \times$ higher for enzyme pairs that share at least one compartment (Table 2, two-sided p-value $=3 \times 10^{-6}$ ), and removing compartment information neglects this information.

To test these competing possibilities, we applied the local and global methods to a network in which compartment information was removed. The yeast metabolic network we used in this study specifies compartments for enzymes and metabolites [10]. We reasoned that identical reactions occurring in different compartments can functionally compensate for each other due to diffusion or transport of metabolites across compartment 

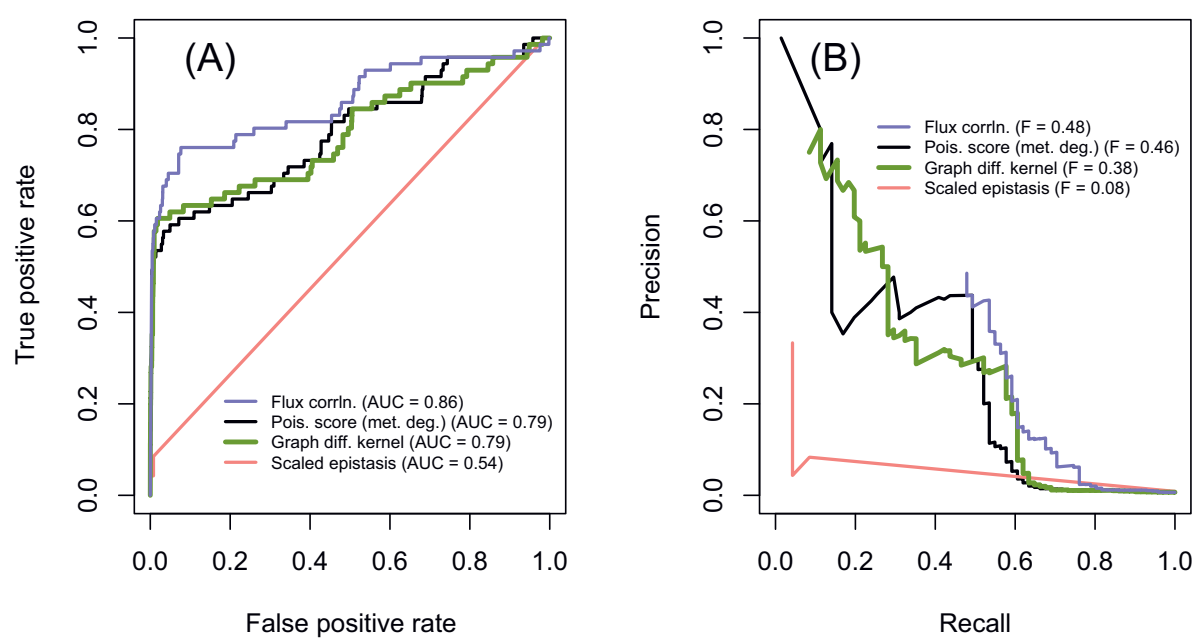

Figure 5 Performance comparison, incorporating metabolic constraints. The best local and global methods are compared with methods that use the knowledge that the network models steady-state metabolism. These methods predict synthetic lethal interactions based on decreases to a fitness function ("Scaled epistasis", ref. [10]) and based on correlations of steady-state fluxes sampled over feasible space ("Flux corrln.", ref. [2]). (A) ROC curve. (B) PR curve.

boundaries. We therefore generated a simplified network that ignores the cellular compartments of the metabolites. Removing the compartments reduced the number of metabolites from 1061 to 646.

The general result for both the local method (Poisson score incorporating metabolite degree) and the global method (graph diffusion kernel with an unweighted network) is that removing compartments improves the precision at high recall at the expense of worse precision at low recall (Figure 6).

\section{Assessment based on database annotations}

We used published methods to assess the ability of different ranking methods to identify enzyme pairs with similar database annotations [11]. This assessment tests the significance of the hypothesis that the average coupling score between all pairs of genes associated with a

Table 1 Compartment distribution of reactions

\begin{tabular}{lr}
\hline Compartment & Number of reactions \\
\hline Cytosol & 599 \\
\hline Mitochondria & 154 \\
\hline Extracellular & 122 \\
\hline Peroxisome & 58 \\
\hline Nucleus & 13 \\
\hline Golgi & 6 \\
\hline Endoplasmic reticulum & 4 \\
\hline Vacuole & 2 \\
\hline Associated with 2 or more compartments & 308
\end{tabular}

Reactions which have metabolites localized to more than one compartment are counted only once and contribute to the total in "Associated with 2 or more compartments".
GO term is higher than between pairs of genes associated with different GO terms. All 835 GO terms mapping to at least 5 and less than 100 enzymes in the network were considered, comprising 538 biological process (BP), 209 molecular function (MF), and 88 cellular compartment (CC). If the null is rejected by the Benjamini-Hochberg procedure at a starting $\mathrm{p}$-value of $0.05 /$ (number of GO terms tested) [12], then the GO term is termed consistent. The fraction of consistent GO terms was computed by this procedure for each method. The assessment was performed for the complete compartment-based network, the reduced compartment-based network with enzymes with negligible fluxes removed, and the network with compartments removed. The category size of 5-100 matches the original publication. Assessment with category sizes of 2-100 and 2-200 yield similar results, but with more categories overall [Additional file 2].

Of all the methods, the graph diffusion kernel clearly performs best overall in producing consistent GO annotations (Table 3). The performance of the methods generally tracks the assessment based on synthetic lethal interaction prediction. Of the local methods, the raw count of shared metabolites performs worst; better are the improved methods that account for enzyme degree;

Table 2 Synthetic lethality and shared compartments

\begin{tabular}{lrr}
\hline & SL & Non-SL \\
\hline Share & 149 & 199,644 \\
\hline Do not share & 21 & 81,061 \\
\hline
\end{tabular}

The category "Share" comprises enzyme pairs that have at least one shared compartment based on metabolite localizations (but do not necessarily share a metabolite). The category "Do not share" contains all other pairs. 

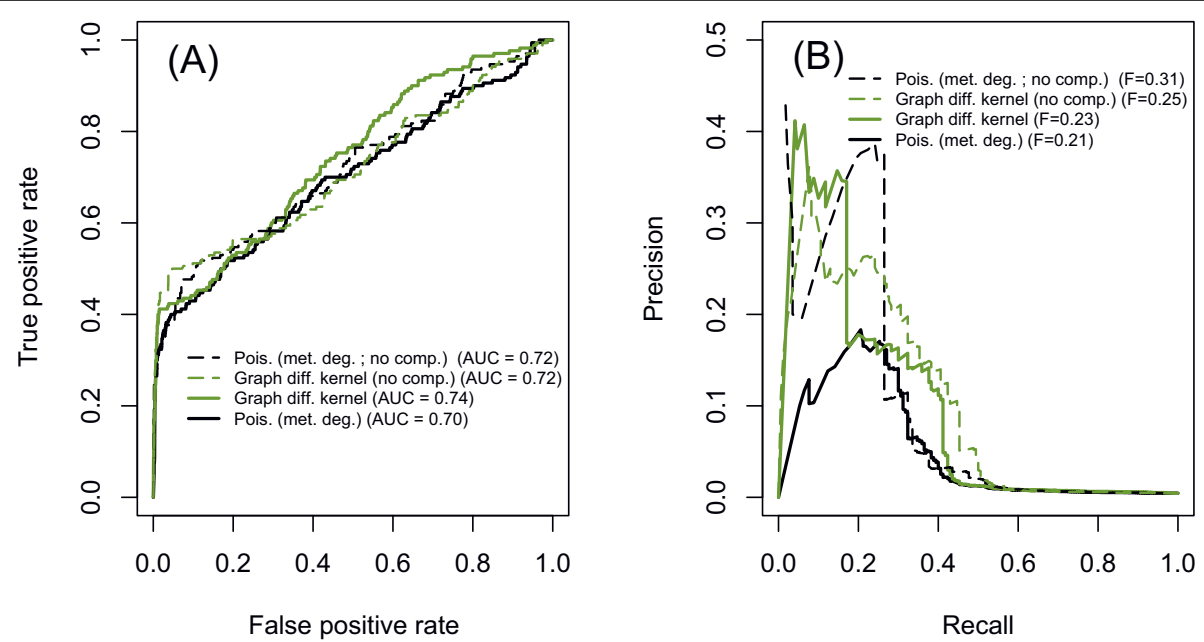

Figure 6 Performance comparison, compartments. Local and global methods are tested for the original metabolic network, and for a reduced network in which metabolite compartment information has been removed. (A) ROC curve. (B) PR curve.

and best of all is the new method that accounts for metabolite degree as well. The graph diffusion method also out-performs the flux sampling method.

We investigated whether the models with or without compartments perform better using a binomial test under the null hypothesis that each of the two models has equal probability of performing better for a particular method. Tests were performed separately for AUC/ ROC, PR/F-score, and the three GO categories, and separately for the full and reduced network. In all cases except GO/Cellular Compartment, two-tailed tests showed no significant deviations from the null hypothesis at $\mathrm{p}=0.05$. The test for Cellular Compartment is significant $(p=0.0396)$, with improved consistency for models that include compartment information.

Flux correlation performs best in the Biological Process category. A surprising result here is that overall flux correlation performs worse than GDK and Poisson (metabolite degree, with or without metabolite associations) with only $70 \%$ consistent GO terms. It is possible the flux correlation method could be improved with longer runs that reduce the statistical noise in the correlations, although the flux calculations already require over $100 \times$ more computer time than any of the other methods (see Computational cost below).

The different methods generate a similar set of consistent $\mathrm{GO}$ term, and terms missed by the graph diffusion kernel are almost always missed by the other methods as well (Figure 7). For cellular compartment, most annotation terms $(72 \%)$ are consistent by all three methods. In the case of biological process and molecular function only $50 \%$ and $71 \%$ of all the annotations are captured by all the three methods.

\section{Discussion}

\section{Performance of local, global, and flux-based methods}

We have investigated the performance of three classes of methods for predicting functional associations in metabolic networks: (i) local methods, based primarily on the metabolites shared by two metabolic enzymes; (ii) global methods, based on the probability that a random walk started at one enzyme will visit a second enzyme; (iii) flux-based methods that use flux balance to identify enzymes with correlated fluxes. The local and global methods are fast and generally applicable to other types of networks, whereas the flux-based methods are computationally expensive and dedicated to metabolism (or other networks that have similar conservation-ofmass constraints). In terms of performance in predicting functional associations, however, the dedicated fluxbased methods have typically been superior. Developing fast methods with performance similar to expensive flux-based methods has been a challenge.

Previous local and global methods have had difficulties with high-degree metabolites. For local methods, metabolites such as water and ATP are often shared by enzymes with very different functions. For global methods, these metabolites introduce many short paths through the network. Often, high-degree metabolites are removed from a network prior to analysis. This approach is undesirable because it introduces an ad hoc tuning parameter, which can lead to over-fitting, and it excludes potentially interesting metabolites from the analysis.

The hypothesis motivating this work is that the difficulties from high-degree metabolites arise from an implicit assumption of a narrow metabolite degree distribution, as opposed to the known long-tailed degree 
Table 3 Performance summary statistic (AUC and F-score) and percentage consistent GO terms

\begin{tabular}{|c|c|c|c|c|c|c|c|c|c|c|c|c|}
\hline \multirow{3}{*}{ Scores } & \multirow{2}{*}{\multicolumn{2}{|c|}{$\begin{array}{l}\text { Performance on } \\
170 \mathrm{SL} \text { pairs } \\
\text { (full network) }\end{array}$}} & \multirow{2}{*}{\multicolumn{2}{|c|}{$\begin{array}{l}\text { Performance on } \\
69 \text { SL pairs } \\
\text { (reduced network) }\end{array}$}} & \multicolumn{8}{|c|}{ Percentage of consitent GO terms } \\
\hline & & & & & \multicolumn{4}{|c|}{$\begin{array}{l}\text { Genes in full network, } \\
283 \mathrm{GO} \text { terms } \\
\text { (152-BP; 85-MF; 46-CC) }\end{array}$} & \multicolumn{4}{|c|}{$\begin{array}{l}\text { Genes in reduced } \\
\text { network, 203 GO terms } \\
\text { (110-BP; 60-MF; 33-CC) }\end{array}$} \\
\hline & $\begin{array}{l}\text { AUC } \\
\text { (ROC) }\end{array}$ & $\begin{array}{l}\text { F-Score } \\
\text { (PR) }\end{array}$ & $\begin{array}{l}\text { AUC } \\
\text { (ROC) }\end{array}$ & $\begin{array}{l}\text { F-Score } \\
\text { (PR) }\end{array}$ & Total & $\mathrm{BP}$ & MF & $\mathrm{CC}$ & Total & BP & MF & $\mathrm{CC}$ \\
\hline \multicolumn{13}{|l|}{ Compartments } \\
\hline Shared Mets & 0.69 & 0.16 & 0.75 & 0.26 & 61 & 52 & 69 & 78 & 50 & 41 & 62 & 61 \\
\hline $\begin{array}{l}\text { Shared Mets } \\
\text { (low degree) }\end{array}$ & 0.67 & 0.15 & 0.76 & 0.27 & 65 & 64 & 61 & 74 & 55 & 58 & 45 & 61 \\
\hline $\begin{array}{l}\text { Hypergeometric } \\
\text { p-value }\end{array}$ & 0.69 & 0.21 & 0.76 & 0.43 & 72 & 64 & 79 & 85 & 63 & 55 & 75 & 67 \\
\hline Bayes Score & 0.70 & 0.21 & 0.76 & 0.43 & 72 & 64 & 79 & 83 & 63 & 55 & 75 & 67 \\
\hline Poisson Score & 0.70 & 0.20 & 0.76 & 0.40 & 72 & 64 & 79 & 85 & 60 & 52 & 73 & 64 \\
\hline Poisson Score (met. degree) & 0.70 & 0.21 & 0.79 & 0.47 & 81 & 73 & 94 & 83 & 74 & 65 & 90 & 73 \\
\hline Graph diffusion kernel score & 0.74 & 0.23 & 0.79 & 0.39 & 94 & 90 & 99 & 98 & 92 & 87 & 97 & 100 \\
\hline Graph diffusion kernel score (weighted edges) & 0.72 & 0.22 & 0.79 & 0.39 & 93 & 88 & 99 & 98 & 92 & 87 & 97 & 100 \\
\hline Flux Correlation & - & - & 0.87 & 0.49 & - & - & - & - & 64 & 66 & 53 & 73 \\
\hline Poisson Score (met. associations, deg.) & 0.70 & 0.21 & 0.79 & 0.47 & 81 & 73 & 93 & 83 & 74 & 65 & 92 & 70 \\
\hline Scaled epistasis & - & - & 0.54 & 0.08 & - & - & - & - & - & - & - & - \\
\hline \multicolumn{13}{|l|}{ No Compartments } \\
\hline Shared mets. & 0.68 & 0.09 & 0.73 & 0.22 & 55 & 47 & 69 & 54 & 49 & 38 & 67 & 52 \\
\hline $\begin{array}{l}\text { Shared mets. } \\
\text { (low deg. mets.) }\end{array}$ & 0.66 & 0.18 & 0.73 & 0.26 & 71 & 72 & 76 & 54 & 64 & 67 & 62 & 58 \\
\hline $\begin{array}{l}\text { Hypergeometric } \\
\text { P-value }\end{array}$ & 0.69 & 0.13 & 0.76 & 0.39 & 66 & 59 & 78 & 65 & 57 & 45 & 73 & 67 \\
\hline Bayes Score & 0.69 & 0.13 & 0.76 & 0.39 & 66 & 59 & 78 & 65 & 57 & 45 & 73 & 67 \\
\hline Poisson Score & 0.69 & 0.12 & 0.75 & 0.33 & 64 & 57 & 76 & 65 & 56 & 44 & 73 & 64 \\
\hline $\begin{array}{l}\text { Poisson Score } \\
\text { (met. degree) }\end{array}$ & 0.72 & 0.31 & 0.80 & 0.49 & 78 & 72 & 93 & 72 & 75 & 65 & 92 & 76 \\
\hline $\begin{array}{l}\text { Graph diffusion } \\
\text { kernel score }\end{array}$ & 0.72 & 0.26 & 0.79 & 0.38 & 94 & 91 & 99 & 93 & 91 & 85 & 98 & 94 \\
\hline $\begin{array}{l}\text { Graph diffusion kernel score } \\
\text { (weighted edges) }\end{array}$ & 0.70 & 0.25 & 0.79 & 0.4 & 93 & 90 & 99 & 93 & 89 & 86 & 93 & 88 \\
\hline Poisson score(met. associations, deg.) & 0.72 & 0.30 & 0.79 & 0.48 & 80 & 74 & 93 & 72 & 75 & 65 & 90 & 79 \\
\hline
\end{tabular}

The best performance in each category is highlighted in bold.

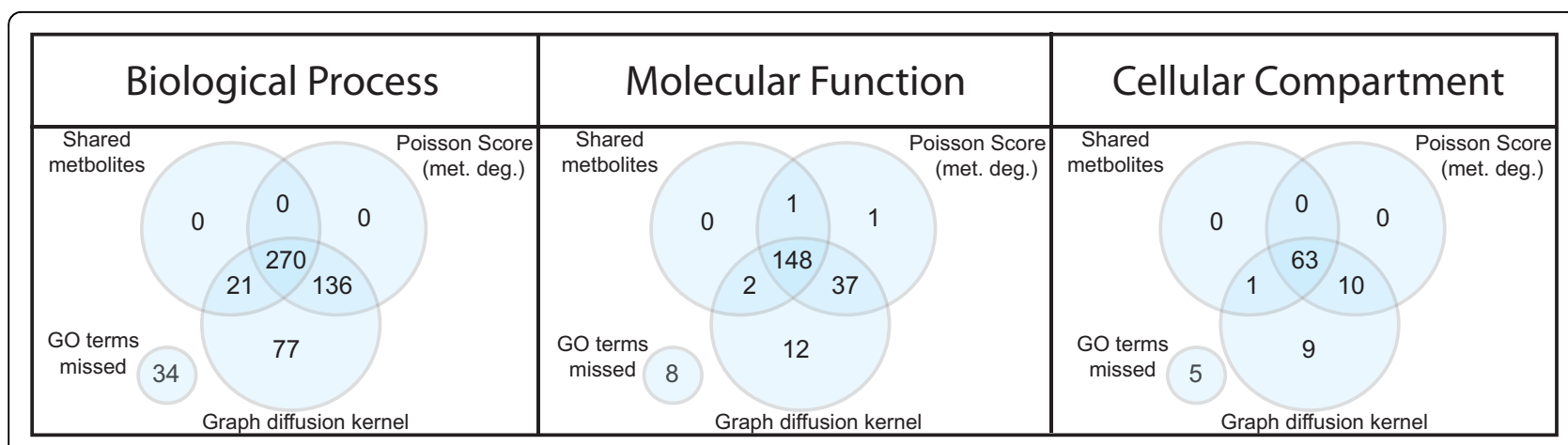

Figure 7 Consistency of Gene Ontology annotations. Venn diagrams indicate the number of consistent Gene Ontology (GO) terms identified for Biological Process, Molecular Function, and Cellular Compartment annotations. GO terms that are inconsistent in all three methods are shown as 'GO terms missed'. 
distribution. The hypergeometric distribution for shared metabolites corrects for enzyme degree, but not for metabolite degree. Consequently a high-degree metabolite such as water is given the same weight as a lowdegree metabolite when counting shared metabolites. Intuitively, high-degree metabolites should be downweighted. Our improved local method uses the known enzyme and metabolite degrees to generate a degreecorrected score with excellent performance.

The global method we examine, a graph diffusion kernel on the bipartite enzyme-metabolite network, also includes a degree normalization that down-weights the contribution of high-degree metabolites (and highdegree enzymes). This method is somewhat more expensive than the local methods, requiring a full matrix inverse rather than sparse matrix multiplication.

The graph diffusion kernel explores the topology of the metabolic network using random walks that visit metabolites and enzymes. Enzyme-metabolite edges are treated as undirected, permitting random walkers to traverse both directions even for a unidirectional reaction. There are no constraints on the flux of random walkers through any enzyme, and the stoichiometry of a metabolite as a reactant or product is ignored.

Flux-balance methods go beyond graph diffusion by adding constraints specific to metabolic networks. Enzyme fluxes are coupled by mass balance and reaction stoichiometry, and correlations between enzyme fluxes can propagate through the network. These additional constraints capture more of the biological reality of metabolism than either shared metabolites or graph diffusion. Predictive performance is also better, presumably because of the biological constraints. A curious point is that flux sampling, with a uniform sample over the feasible space, performs better than calculations of epistatic effects based on reductions to an optimized fitness objective function. This may indicate errors in the assumed objective function for cellular fitness. The main drawback of flux-balance methods is the high computational cost.

In summary, graph diffusion methods have become a method of choice for analyzing many types of networks. While the degree-corrected local methods provide a substantial improvement over previous local methods, the graph diffusion kernel using the entire network topology performs somewhat better. Dedicated fluxsampling methods are slightly better for predicting genetic interactions, but take over $100 \times$ longer to calculate and are much more difficult to implement.

\section{Disagreement between network-based predictions and database annotations}

As the graph diffusion kernel (GDK) method provides a good trade-off between performance (assessed by genetic interaction prediction) and computational efficiency, it is likely to be a method of choice. The average semantic similarity for gene pairs highly ranked by the GDK provides an additional assessment of performance (Figure 8). GDK scores of 10 and more have an average semantic similarity of 4 or greater, corresponding to no more than 116 genes annotated to the parent category. Thus, on average, a high GDK score indicates a similar database annotation.

These average results, however, do not always hold for individual gene pairs. A cross-tabulation of semantic similarity vs. GDK score demonstrates that many gene pairs with high GDK scores nevertheless have essentially no semantic similarity (Figure 9). These GDK predictions of functional association would essentially be scored as false-positive predictions based on the lack of similarity in database annotations. Because of the overall good performance of the GDK method, we systematically investigated the most extreme cases in an attempt to suggest a reason for the disagreement.

We therefore selected examples with high GDK scores and low semantic similarity. A GDK threshold of score $\geq 50$ focused attention on the top-ranked GDK pairs, where on average the semantic similarity corresponds to only 23 genes annotated to the parent category of a pair. We then set a threshold of semantic similarity $\leq 2$. This value is substantially below the semantic similarity of gene pairs selected at random, and was chosen to yield a number of example that was feasible for case-bycase analysis, a data set of 101 pairs denoted putative false positives [Additional file 3].

Manual inspection suggests that these cases can be classified into 4 main categories: unannotated, transport-synthesis, pathway-boundary, and secondary activity (Table 4). The unannotated category comprises $10 \%$ of the cases and indicates that a gene lacks database annotations for Biological Process, Cellular Component, and Metabolic Function, despite being included in a metabolic reconstruction. The secondary activity category, with only 3 cases, is a related discrepancy in which the secondary metabolic activity of an enzyme is present in the metabolic reconstruction but not noted in $\mathrm{GO}$.

The transport-synthesis category is the largest, with about $65 \%$ of the cases. In these examples, the GDK predicts an association between enzymes responsible for synthesis and transport (usually extracellular to intracellular) of the same metabolite. Thus, both enzymes are fulfilling the same role of increasing the intracellular concentration of a metabolite. The structure of the GO annotations does not reflect this close functional association, however. Examples include transport of choline/ ethanolime (HNM1-CKI1), allantoate (DAL5-DAL1), sterols (AUS1-ERG27), and uridine (FUI1-URK1). 


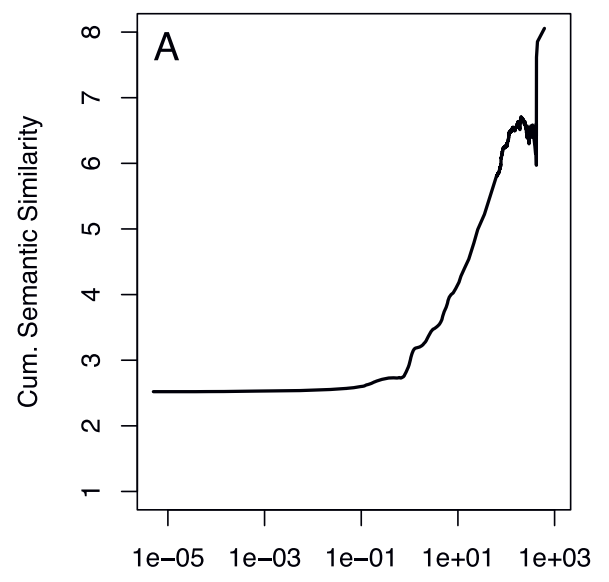

GDK Score

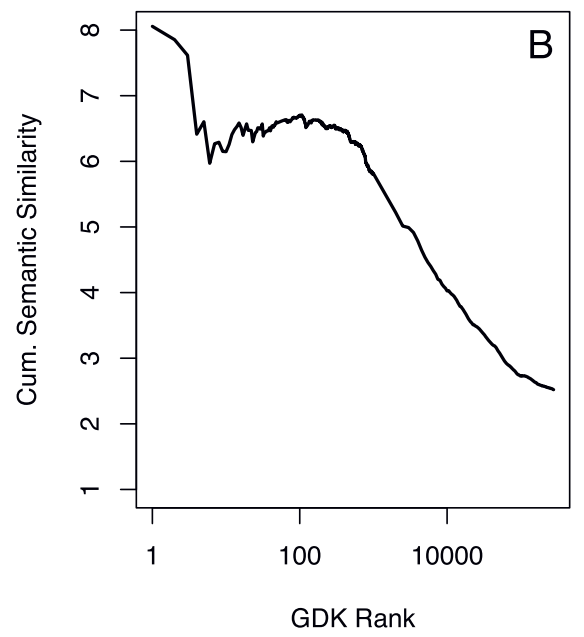

Figure 8 Cumulative semantic similarity. The graph diffusion kernel was used to calculate scores for gene pairs (compartment model, diffusion parameter $\gamma=1$ ). For each gene pair, the semantic similarity was also calculated for Biological Process, Cellular Component, and Molecular Function, and the largest of the three values was retained. The cumulative average of this maximum value was then calculated for GDK thresholds of decreasing stringency. (A) The threshold is shown as the GDK score, from least stringent ( $\mathrm{score}=5 \times 10^{-6}$, the smallest GDK score) to most stringent (617.2, the largest GDK score). (B) The threshold is shown as the rank order, from most stringent $($ rank $=1)$ to least stringent (rank $=279,000$, the total number of pairs).

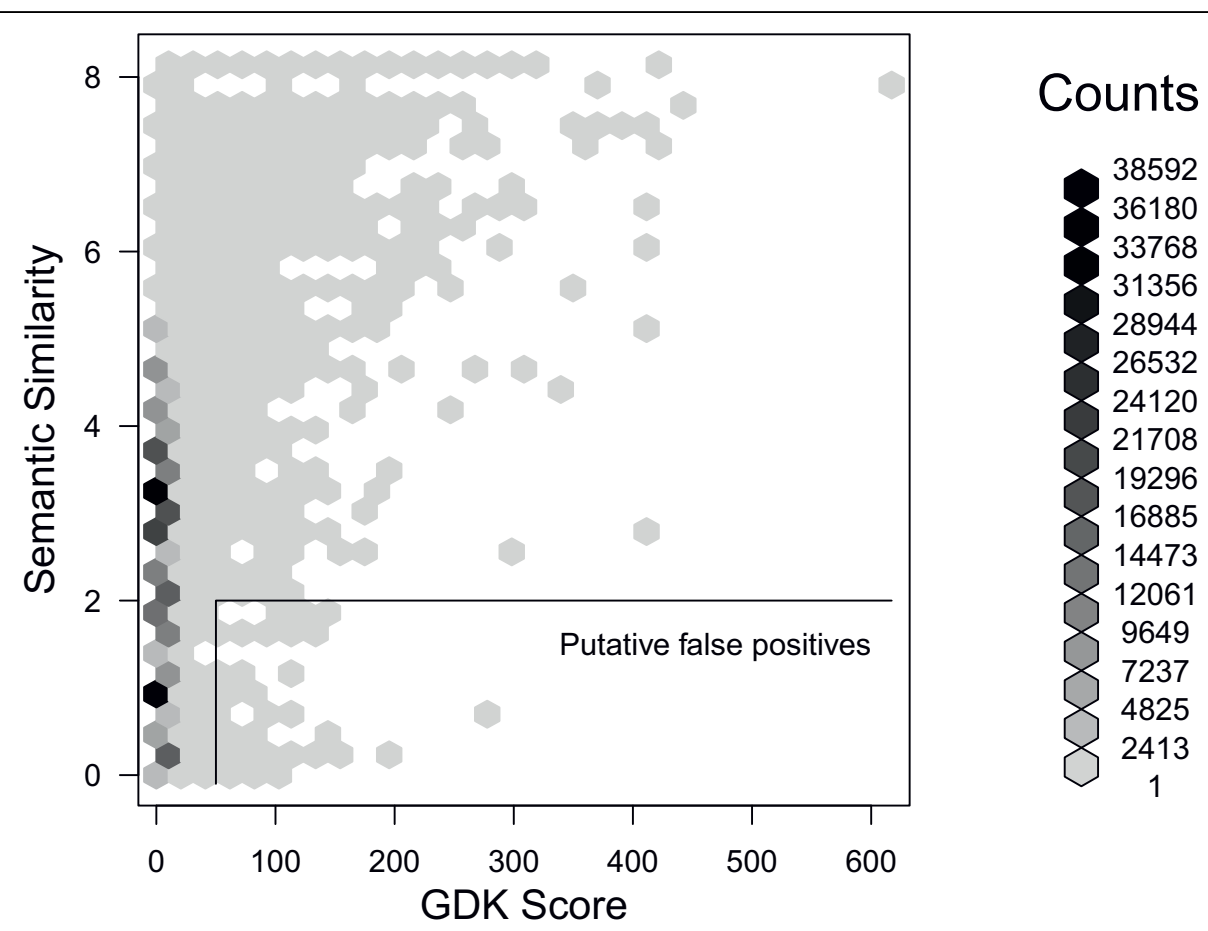

Figure 9 Putative false positive functional associations. A density plot of gene pairs binned by semantic similarity and graph diffusion kernel (GDK) score indicates putative false positive functional associations. The semantic similarity for each gene pair was calculated as the maximum of the values for Biological Process, Cellular Component, and Molecular Function. The average of this value over all gene pairs is 2.52, and gene pairs with semantic similarity below this value have essentially no semantic similarity. Gene pairs with GDK scores of 50 and above have an average semantic similarity of 5.6, corresponding to 23 genes annotated to the parent category. Putative false positives were defined for exploratory purposes as having GDK scores of 50 and above but semantic similarity of 2 or below, corresponding to 850 genes annotated to the parent category. The putative false-positive region is indicated and contains 101 gene pairs. 
Table 4 Categories of gene pairs with high network association and low semantic similarity.

\begin{tabular}{lr}
\hline Category & $\begin{array}{r}\text { Number of Gene Pairs } \\
\text { (of 101) }\end{array}$ \\
\hline Transport-Synthesis & 66 \\
\hline Unannotated & 12 \\
\hline Pathway boundary, Quinone metabolism & 7 \\
\hline \hline Pathway boundary, Glycolysis & 4 \\
\hline Secondary activity & 3 \\
\hline Pathway boundary, N-acetylation & 2 \\
\hline Pathway boundary, Purine metabolism & 2 \\
\hline Pathway boundary, TCA & 2 \\
\hline Pathway boundary, Fumarate metabolism & 1 \\
\hline Pathway boundary, Glycoprotein synthesis & 1 \\
\hline Pathway boundary, Redox & 1 \\
\hline
\end{tabular}

The final category, pathway-boundary, arises when the boundary between two well-accepted pathways cuts through a metabolite. Enzymes that connect to this metabolite are then annotated to very different pathways, despite a close network-level association. These cases are responsible for $20 \%$ of the total. Examples include associations between enzymes in the TCA cycle and those using TCA metabolites for amino acid synthesis, enzymes with different roles in glycoprotein synthesis, and enzymes responsible for quinone metabolism [Additional file 3].

\section{Conclusions}

In analyzing large networks, it has become common to delete high-degree vertices. This practice is questionable. It depends on an arbitrary high-degree cutoff, usually without any clear break in a vertex degree distribution. It can remove vertices that are of interest, and it can introduce unknown biases into the analysis.

Here we have introduced methods that are readily applied to networks with high-degree hubs. Local methods use known degree distributions to correct for highdegree enzymes and metabolites, and global methods use graph diffusion kernels to rank the association between pairs of enzymes. We show that these methods outperform previous methods that eliminate high-degree vertices from the networks. The context is cellular metabolism, where high-degree metabolites like water and ATP are shared by many enzymes. Our methods are able to infer functional associations between enzymes, without being misled by sharing of these high-degree metabolites.

In several cases, enzymes predicted by network analysis to have high functional association have very little similarity in database annotations. Some of these cases are due to a discrepancy between the metabolic reconstruction, which records a reaction for an enzyme, and the annotation database, which lacks information or omits a secondary activity. Two additional patterns were observed, however, which relate to the structure of Gene Ontology hierarchies. First, enzymes that are responsible for synthesis and transport of the same metabolite often have little annotation similarity. Second, conventional pathway definitions may place two enzymes with strong network-level associations on opposite sides of a pathway boundary.

The methods developed here should be applicable in general to other bipartite networks, particularly those with high-degree hubs.

\section{Methods}

\section{Yeast Metabolic network reconstruction}

The Yeast metabolic network used in our study was obtained from the database maintained by systems biology group, University of California, San Diego [10,13]. The file "Sc_iND750_GlcMM.xml" corresponding to the minimal media condition was obtained from http://gcrg. ucsd.edu/Downloads/Cobra_Toolbox. This network has 1061 metabolites, 1266 reactions and 750 genes. The stoichiometry matrix $S(m, r)$ provides the number of metabolites $m$ consumed or produced in reaction $r$. The reaction-gene association matrix $E(r, e)$ in the metabolic network indicates whether reaction $r$ can be catalyzed by enzyme $e$.

Coupling measures based on metabolic bipartite network A bipartite network has two disjoint sets of vertices with edges only between vertices of different sets. In the case of the metabolic network, we consider enzymes $e$ and the metabolites $m$ as disjoint vertices in a bipartite graph. We use various metabolic coupling measures between two enzymes in this graph to predict synthetic lethal genetic interactions. Towards this goal, we use both methods from literature (based on shared metabolites [6], shared metabolites after removing high degree metabolites $[3,4])$ and other methods proposed here.

\section{Shared metabolite count (with and without hubs)}

The coupling between metabolites can be calculated using the stoichiometry matrix as $\hat{S}_{S}^{T}$ [6]. The elements of $\hat{S}$, denote the participation of a metabolite $i$ in a reaction $j$ with a value 1 and 0 otherwise ( $\hat{S}$ is binary version of the stoichometry matrix, S). This idea extended to the coupling of genes based on the bipartite metabolic network could be represented as $\hat{M}_{\hat{M}}{ }^{T}$, where $M^{T}=\hat{S} E$, and $\hat{M}$ is the binary version of the matrix $M$. The element $C_{i j}$ of the matrix $C$ now represents number of metabolites shared between enzyme $i$ and enzyme $j$. 
The metabolite degree is defined as the number of reactions in which a metabolite participates. In previous work, high-degree metabolites have been excluded from metabolite sharing (equivalent to ignoring the rows corresponding to metabolite hubs in the stoichiometry matrix) [3]. In our calculations of shared metabolites, the top 5\% of metabolites were excluded (53 metabolites participating in 13 or more reactions).

\section{Bayesian score}

We used our previous method based on $2 \times 2$ contingency table for calculating the metabolites shared between two enzymes [2]. Briefly, this measure is obtained as a log likelihood ratio of alternative to null hypothesis. Under the null, the probability of connection of a metabolite to both enzymes is product of the individual probabilities of connections. Let $n, n_{1}, n_{2}$ and $n_{12}$ represent the total number of metabolites in the network, the number connected to enzyme 1, the number connected to enzyme 2 , and the number connected to both. The score is then

$$
\begin{aligned}
\text { Bayesian Score }=\log & {\left[\frac{(n+1) \cdot C\left(n, n_{2}\right)}{(n+2) \cdot(n+3) \cdot C\left(n_{1}, n_{12}\right)}\right] } \\
& -\log \left[C\left(n-n_{1}, n_{2}-n_{12}\right)\right] .
\end{aligned}
$$

The combinatorial factor $C(n, k)$ is $n ! / k !(n-k)$ !. This score increases when $n_{12}$ is either larger or smaller than the value $n_{1} n_{2} / n$ expected under the null hypothesis (analogous to a two-sided test). For $n_{12}$ smaller than the null expectation, we used $\operatorname{score}\left(n_{12}\right)-\mid \operatorname{score}\left(n_{12}\right)$ - score $\left(n_{1} n_{2} / n\right) \mid$ to restrict attention to enrichment.

\section{Hypergeometric p-value score}

The score based on the hypergeometric $\mathrm{p}$-value for characterizing the metabolic coupling between the enzymes 1 and 2 is

$$
\begin{aligned}
& \text { Hypergeometric } \mathrm{p} \text { - value score } \\
& \qquad=-\log \left[\sum_{k=n_{12}}^{\min \left(n_{1}, n_{2}\right)} \frac{C\left(n_{1}, k\right) \cdot C\left(n-n_{1}, n_{2}-k\right)}{C\left(n, n_{2}\right)}\right] .
\end{aligned}
$$

\section{Poisson score}

This score is also obtained as the log-likelihood ratio of an alternative to null model for the observed number of shared partners. Both the alternative and the null employ a Poisson distribution with a single parameter $\lambda$. For the alternative, $\lambda_{\text {alt }}=n_{12}$, the observed count; for the null, $\lambda_{\text {null }}=n_{1} n_{2} / n_{\text {tot }}$. The total number of metabolite-enzyme edges is $n_{\text {tot }} ; n_{1}$ and $n_{2}$ are the numbers of metabolites connected to each enzyme; and $n_{12}$ is the intersection of the metabolites in $n_{1}$ and $n_{2}$. The Poisson score is

$$
\begin{aligned}
\text { Poisson Score } & =n_{12} \cdot \log \left[\frac{n_{12}}{n_{1} \cdot n_{2} / n_{\text {tot }}}\right] \\
& -\left|n_{12}-\frac{n_{1} \cdot n_{2}}{n_{\text {tot }}}\right| .
\end{aligned}
$$

The absolute value for the second term in Eq. 3 ensures that large scores come from enrichment rather than depletion of shared metabolites.

\section{Poisson score with metabolite degree}

The null model in the Poisson score was further improved by considering a different value for $\lambda_{\text {null }}$ using the degree distribution of the metabolites connected to the enzymes. Let $k_{i 1}, k_{i 2}, \ldots, k_{i n_{i}}$ be the degree of the metabolites $m_{i 1}, m_{i 2}, \ldots, m_{i n}$ connected to enzyme $i$ in the enzyme pair $(i=1,2)$. The probability of the metabolite $m_{11}$ to be connected to enzyme 2 is

$$
\begin{aligned}
\operatorname{Pr}\left(m_{11} \sim \text { Enzyme } 2\right) & =1-\left[1-\frac{n_{2}}{n_{\text {tot }}}\right]^{k_{11}} \\
& \approx 1-\exp \left[-\frac{k_{11} \cdot n_{2}}{n_{\text {tot }}}\right] .
\end{aligned}
$$

The average number of metabolites connected to enzyme 1 that are also connected to enzyme 2 is then

$$
\lambda_{\text {null }}^{12}=\sum_{i=1}^{n_{1}}\left[1-\exp \left(-\frac{k_{1 i} \cdot n_{2}}{n_{\text {tot }}}\right)\right] \text {. }
$$

The values of $\lambda_{\text {null }}^{12}$ and $\lambda_{\text {null }}^{21}$ are in general different due to different degree distribution of the metabolites connected to enzymes 1 and 2 . The value of $\lambda_{\text {null }}$ used in the improved null model is obtained by arithmetic mean of $\lambda_{\text {null }}^{12}$ and $\lambda_{\text {null }}^{21}$. The final expression for the improved Poisson score is

$$
\begin{aligned}
& \text { Poisson Score }(\text { met. degree }) \\
& \qquad \begin{array}{l}
=n_{12} \cdot \log \left[\frac{n_{12}}{0.5 \cdot \lambda_{\text {null }}^{12}+0.5 \cdot \lambda_{\text {null }}^{21}}\right] \\
-\left|n_{12}-0.5 \cdot \lambda_{\text {null }}^{12}-0.5 \cdot \lambda_{\text {null }}^{21}\right| .
\end{array}
\end{aligned}
$$

\section{Poisson score with metabolite associations and degree} A further improved Poisson score can be generated using a more elaborate $\lambda_{\text {null, }}$ accounting for the full 
metabolite degree distribution. The probability of $k$ connections given the status of the metabolites $m_{1}, \ldots, m_{\mathrm{i}}$ connected to enzyme $E, P^{E}\left(k \mid m_{1}, \ldots, m_{i}\right)$ is

$$
\begin{aligned}
P^{E} & \left(k \mid m_{1}, \ldots, m_{i}\right) \\
& =P^{E}\left(k \mid m_{1}, \cdots, m_{i-1}\right) \cdot P\left(E \nsim m_{i} \mid k\right) \\
& +P^{E}\left(k-1 \mid m_{1}, \ldots, m_{i-1}\right) \cdot P\left(E \sim m_{i} \mid k-1\right) .
\end{aligned}
$$

The probability of enzyme connected and not connected to the metabolite $m_{i}$ are estimated as

$$
\begin{gathered}
P\left(E \sim m_{i} \mid k-1\right)=1-\exp \left(-\frac{k_{i} \cdot\left(n_{E}-k+1\right)}{n_{\mathrm{tot}}}\right) \\
P\left(E \nsim m_{i} \mid k\right)=\exp \left(-\frac{k_{i} \cdot\left(n_{E}-k\right)}{n_{\mathrm{tot}}}\right) .
\end{gathered}
$$

We take $P^{E}\left(k=-1 \mid m_{1}, \ldots, m_{i}\right)=0, P^{E}(k=0 \mid-)=1$ in the calculations and obtain $P^{E}\left(k \mid m_{1}, \ldots, m_{i}\right)$ for the enzymes $\mathrm{A}$ and $\mathrm{B}$ in the pair (and respectively connected to $n_{1}$ and $n_{2}$ metabolites) to obtain the $\lambda_{\text {null }}$,

$$
\begin{aligned}
\lambda_{\text {null }}= & 0.5 \cdot \sum_{k=0}^{\min \left(n_{1}, n_{2}\right)} k \cdot P^{A}\left(k \mid m_{1}, \ldots, m_{n_{1}}\right) \\
& +0.5 \cdot \sum_{k=0}^{\min \left(n_{1}, n_{2}\right)} k \cdot P^{B}\left(k \mid m_{1}, \ldots, m_{n_{2}}\right) .
\end{aligned}
$$

We use $\lambda_{\text {null }}$ (Eq. 9) to obtain the Poisson score that takes metabolite associations and degree into account,

$$
\begin{aligned}
\text { Poisson Score } & \text { (met. association, degree) } \\
& =n_{12} \cdot \log \left[\frac{n_{12}}{\lambda_{\text {null }}}\right]-\left|n_{12}-\lambda_{\text {null }}\right| .
\end{aligned}
$$

Results from this more complicated model are included in Table 1, but not discussed in the text as the method is more complicated yet performs no better than simpler Poisson score methods.

\section{Graph diffusion kernel}

Graph diffusion kernels are solutions to the steady-state density distribution for continuous-time random walk or diffusive process on a graph with sources and sinks [8]. The adjacency matrix for the calculation of the GDK measure (Eq. 11) has a block structure whose dimension is given by the sum of number of metabolites and enzymes in the yeast metabolic network (i.e. $1061+750=1811$ ),

$$
A=\left[\begin{array}{cc}
0 & \hat{M}^{T} \\
\hat{M} & 0
\end{array}\right] .
$$

The matrix $\hat{M}$ is the binary version of the matrix $M$ defined as $M^{T}=\hat{S} E$ and captures the direct links from metabolites to enzymes. The degrees of the nodes are summarized by the diagonal matrix $\mathrm{D}\left(\mathrm{D}_{i i}=\sum_{\mathrm{j}} \mathrm{A}_{\mathrm{ij}}\right.$, with $A_{i j}$ the elements of the adjacency matrix, A). The graph diffusion kernel score with normalization for the node degrees is

$$
\begin{aligned}
& \text { Graph diffusion kernel score } \\
& \qquad=\left[(1+\gamma) \cdot I-D^{-\frac{1}{2}} A D^{-\frac{1}{2}}\right]^{-1} .
\end{aligned}
$$

The parameter $\gamma$ controls the extent of diffusion, or equivalently the length of the random walks. These lengths are distributed exponentially, with the probability of a $d$-step walk proportional to e- ${ }^{\gamma d}$. The results shown in this work are for a value of $\gamma=1$. Results were not sensitive to the value of $\gamma$, with similar results over a range from 0.5 to 120 . The entries in the kernel corresponding to the enzyme-enzyme relationships were then extracted to predict genetic interactions. For readability, GDK scores displayed in the figures are multiplied by $10^{4}$.

\section{Graph diffusion kernel with weighted edges}

We also considered a version of the graph diffusion kernel with weighted edges. Here we used the full version of the matrix $M\left(M^{T}=\hat{S} E\right)$ in the adjacency matrix $A$ rather than a binary version as used in the non-weighted case (Eq. 11). The element $\left(M^{T}\right)_{i j}$ of the matrix $M^{T}=$ $\hat{S}^{E}$ represents the number of times a metabolite $i$ is associated with the enzyme $j$ through various reactions. Then kernel score with weighted edges is obtained using the same procedure as described above. Results are shown for $\gamma=1$ and remained the same for higher $\gamma$ values.

\section{Scores without compartments}

The model obtained from the BIGG database is a fully compartmentalized model with same metabolites localized to different compartments represented separately. Some metabolites may move between compartments freely, others through ion-channels by chemical gradients or through transporters. This may bring the reactions that use the metabolites that diffuse freely in different compartments closer in that they share either the substrates or products. To investigate the effect of this in our analysis, we combined same metabolites localized to different compartments (by adding the rows of the stoichometric matrix). Then we calculated all the metabolic coupling measures (except enzyme flux correlation measure) with the compartment-free metabolic 
network. There were 646 metabolites in the compartment-free network.

\section{Enzyme flux correlation}

The performance of various scores considered in this work were compared with enzyme flux correlation score used in our previous study [2]. Briefly, the method is based on feasible reaction fluxes obtained under stoichometric and reaction flux constraints at steady state [14]. The set of feasible reaction fluxes were sampled using a Markov random sampling algorithm under in silico medium similar to YPD [15]. Then reaction fluxes were transformed to enzyme fluxes. The enzyme flux correlation between two enzymes is then obtained by calculating the Pearson correlation coefficient over the various enzyme flux samples. The details of the calculations are available elsewhere [2]. This entire procedure from random sampling to calculating correlations was repeated 3 times with different random seeds, with no evidence of non-ergodic sampling among the three runs. Final predictions used absolute value of correlation averaged over three runs. A preliminary step before random sampling removes all blocked reactions which carry no flux. The reduced model used for flux sampling had 477 metabolites, 582 reactions and 469 enzymes. The flux sampling procedure was carried out with the COBRA MATLAB toolbox [16]. The absolute value of the flux correlation is used for ranking.

\section{Scaled epistasis}

The scaled epistasis values corresponding to the minimal media were obtained from a previous study [9]. The file "fitness_data_nominal.txt" containing the fitness of insilico single and double gene yeast knockouts were obtained from http://kishony.med.harvard.edu/prism/ index.html. For calculating the AUC and F-score from scaled epistasis score, only enzyme pairs with epistasis score were considered. We did not calculate GO consistency scores for this method because it performed poorly for predicting genetic interactions.

\section{Synthetic lethality data sources}

Synthetic lethality data for this study was obtained from the BioGRID database (version 2.0.46) [17]. There were 97 synthetic lethal and 73 synthetic growth defect interactions in the BioGRID database that had both the genes in the yeast metabolic network. There were 39 synthetic lethal and 30 growth deflect interactions in the BioGRID database that had both the genes in the reduced model used in the flux sampling procedure.

\section{Performance metrics}

The ROC curves, AUC, F-score and PR curves were generated in $\mathrm{R}$ using the ROCR package [18]. The ROC and PR curves are shown with a downsampling option in the plot set to 5000 .

\section{Gene Ontology assessment}

We used a procedure proposed in a previous study for validating the functional gene similarity measures [11]. We used gene ontology (GO) annotation terms from all the three categories biological process, cellular compartment and molecular function. A GO term is termed consistent if the average metabolite coupling score between all pairs of metabolic genes associated with the GO term is greater than genes that do not share a same annotation.

The percent of consistent GO terms were calculated for each bipartite coupling measure. We considered only GO terms associated with 5 though 100 genes. For each GO term, the pairwise coupling score between metabolic genes associated with it are averaged. The statistical significance of this averaged score is assessed by random shuffling of gene GO annotation associations, maintaining both the annotation and gene distribution. We calculated an empirical p-value based on 10,000 iterations for each GO term. These empirical p-values were corrected for multiple testing of many GO terms to control for false discovery rates [12]. The consistency score was obtained by the proportion of GO terms that were significant with a false discovery rate of 0.05 . The $\mathrm{GO}$ gene associations of yeast corresponding in the file gene_association.sgd was obtained from the Saccharomyces genome database, http://downloads.yeastgenome. org/literature_curation/.

\section{Semantic Similarity}

Semantic similarity was calculated as $l_{n}\left(N_{T} / N_{P}\right)$, where the total number of genes $N_{T}=6310$ for yeast, and the number of genes annotated to the closest parent category of two genes is $N_{P}[19,20]$. Semantic similarity values were calculated separately for the three main Gene Ontology hierarchies: Biological Process, Cellular Component, and Metabolic Function. These three values were then summarized by the maximum of the three to identify functional associations inferred from network structure that do not match any known annotation similarity.

\section{Computational cost}

The methods proposed in this work are computationally less expensive as compared to the flux correlation based approaches. The computation of flux correlation takes about 15 hours (each sampling run taking about 5 hours). Computing the other scores was performed as a single calculation that required only 9 minutes. The graph diffusion kernel, part of this single calculation, 
was computed directly as the inverse of the graph Laplacian rather than as a repeated matrix multiplication.

\section{Additional material}

Additional file 1: Performance of raw neighbor count. The data from Figure 3 are replotted with the addition of the name, cellular localization ( $c=$ cytoplasm, $\mathrm{m}=$ mitochondrion, $\mathrm{n}=$ nucleus, $\mathrm{x}=$ extracellular), and degree of each metabolites at the position it is removed.

Additional file 2: Consistent GO terms and category size. GO consistency analysis results are provided for category sizes of 2-100 and 2-200, compared with 5-100 in the main text

Additional file 3: Putative false positives. The 101 gene pairs with high network association and low semantic similarity are tabulated and the reasons for disagreement are annotated.

\section{Acknowledgements}

BV and JSB acknowledge funding from NIH R24 DK082840. JSB acknowledges support from NSF CAREER MCB 0546446. We acknowledge help from Yasir Suhail in performing semantic similarity calculations.

\section{Author details}

'Department of Biomedical Engineering, Johns Hopkins University, Baltimore, MD21218, USA. ${ }^{2}$ High-Through put Biology Center, Johns Hopkins School of Medicine, Baltimore, MD21205, USA.

\section{Authors' contributions}

BV and JSB conceived the study and wrote the manuscript. BV performed the work. All authors read and approved the final manuscript

Received: 16 July 2009 Accepted: 14 July 2010 Published: 14 July 2010

\section{References}

1. Inmels J, Levy R, Barkai N: Principles of transcriptional control in the metabolic network of Saccharomyces cerevisiae. Nat Biotechnol 2004, 22:86-92.

2. Veeramani B, Bader JS: Metabolic flux correlations, genetic interactions, and disease. J Comput Biol 2009, 16(2):291-302.

3. Lee DS, Park J, Kay KA, Christakis NA, Oltvai ZN, Barabási AL: The implications of human metabolic network topology for disease comorbidity. Proc Natl Acad Sci USA 2008, 105(29):9880-9885.

4. Kharchenko P, Church GM, Vitkup D: Expression dynamics of a cellular metabolic network. Mol Syst Biol 2005, 1:2005.0016.

5. Notebaart RA, Teusink B, Siezen RJ, Papp B: Co-regulation of metabolic genes is better explained by flux coupling than by network distance. PLoS Comput Biol 2008, 4:e26.

6. Becker SA, Price ND, Palsson BO: Metabolite coupling in genome-scale metabolic networks. BMC Bioinformatics 2006, 7:111.

7. Tsuda K, Noble WS: Learning kernels from biological networks by maximizing entropy. Bioinformatics 2004, 20(Suppl 1):i326-i333.

8. Qi Y, Suhail Y, yi Lin Y, Boeke JD, Bader JS: Finding friends and enemies in an enemies-only network: a graph diffusion kernel for predicting novel genetic interactions and co-complex membership from yeast genetic interactions. Genome Res 2008, 18(12):1991-2004.

9. Segrè D, Deluna A, Church GM, Kishony R: Modular epistasis in yeast metabolism. Nat Genet 2005, 37:77-83.

10. Duarte NC, Herrgård MJ, Palsson BO: Reconstruction and validation of Saccharomyces cerevisiae iND750, a fully compartmentalized genomescale metabolic model. Genome Res 2004, 14(7):1298-1309.

11. Rokhlenko O, Shlomi T, Sharan R, Ruppin E, Pinter RY: Constraint-based functional similarity of metabolic genes: going beyond network topology. Bioinformatics 2007, 23(16):2139-46.

12. Benjamini YHY: Controlling the false discovery rate: a practical and powerful approach to multiple testing. Journal of the Royal Statistical Society 1995, 57:289
13. Schellenberger J, Park J, Conrad T, Palsson B: BiGG: a Biochemical Genetic and Genomic knowledgebase of large scale metabolic reconstructions. BMC Bioinformatics 2010, 11:213.

14. Price ND, Reed JL, Palsson BO: Genome-scale models of microbial cells: evaluating the consequences of constraints. Nat Rev Microbiol 2004, 2(11):886-897.

15. Harrison R, Papp B, Pál C, Oliver SG, Delneri D: Plasticity of genetic interactions in metabolic networks of yeast. Proc Natl Acad Sci USA 2007 104(7):2307-2312.

16. Becker SA, Feist AM, Mo ML, Hannum G, Palsson BO, Herrgard MJ: Quantitative prediction of cellular metabolism with constraint-based models: the COBRA Toolbox. Nat Protoc 2007, 2(3):727-738.

17. Stark C, Breitkreutz BJ, Reguly T, Boucher L, Breitkreutz A, Tyers M: BioGRID: a general repository for interaction datasets. Nucleic Acids Res 2006, , 34 Database: D535-D539.

18. Sing T, Sander $O$, Beerenwinkel $N$, Lengauer $T$ : ROCR visualizing classifier performance in R. Bioinformatics 2005, 21(20):3940-3941.

19. Lord PW, Stevens RD, Brass A, Goble CA: Investigating semantic similarity measures across the Gene Ontology: the relationship between sequence and annotation. Bioinformatics 2003, 19(10):1275-1283.

20. Resnik P: Semantic Similarity in a Taxonomy An Information-Based Measure and its Application to Problems of Ambiguity in Natural Language. Journal of Artificial Intelligence Research 1999, 11:95-130.

\section{doi:10.1186/1752-0509-4-95}

Cite this article as: Veeramani and Bader: Predicting functional associations from metabolism using bi-partite network algorithms. BMC Systems Biology 2010 4:95

\section{Submit your next manuscript to BioMed Central and take full advantage of:}

- Convenient online submission

- Thorough peer review

- No space constraints or color figure charges

- Immediate publication on acceptance

- Inclusion in PubMed, CAS, Scopus and Google Scholar

- Research which is freely available for redistribution
C Biomed Central 